\title{
Automated Characterization of Muscle Architectural Variation in Ultrasound Images
}

\author{
Jan Egil Kirkebø \\ Department of Informatics \\ University of Oslo \\ Oslo, Norway \\ janki@ifi.uio.no
}

\author{
Amelie Werkhausen \\ Norwegian School of Sport \\ Sciences \\ Oslo, Norway \\ ameliew@nih.no
}

\author{
Olivier Seynnes \\ Norwegian School of Sport \\ Sciences \\ Oslo, Norway \\ oliviers@nih.no
}

\author{
Andreas Austeng \\ Department of Informatics \\ University of Oslo \\ Oslo, Norway \\ andrea@ifi.uio.no
}

\begin{abstract}
Fascicle (fiber bundle) orientation and length are useful parameters to infer the force potential of skeletal muscles. Ultrasound imaging is commonly used to determine fascicle characteristics. In most of the literature, automated methods simplify analysis by assimilating fascicles and aponeuroses (connective tissue on which fascicles are anchored) to straight lines that are homogeneously arranged along the muscle length. In practice, manual adjustments are often needed to the realizations of the proposed automated methods due to the low signal-to-noise ratio of the images. We propose a fully automated and robust method that determines non-linear aponeuroses and fascicles over entire panoramic images, reflecting the real structure of the muscle and its fibers.
\end{abstract}

\section{Keywords—ultrasound, muscle, aponeuroses, fascicle}

\section{INTRODUCTION}

For characterization of human muscles, non-invasive 2D ultrasound B-mode images are commonly used. A schematic illustration of muscle imaging is shown in Figure 1. We see that there are several challenges with the imaging process. Firstly, the obtained ultrasound image suffers from a low signal-to-noise ratio, obfuscating the actual structures that are being imaged. Also, the size of the ultrasound transducer limits the field of view of the ultrasound image to a minor portion of the actual muscle. Panoramic scans can be used to overcome the limited field of view but their analysis remains complex.

Manual analysis of ultrasound images can be painstaking and prone to subjective interpretations. Automated methods have been developed for interpretation of fascicles [1], [2]. However, proposed methods often rely on a number of assumptions, like the approximation of the shape of aponeurosis and fascicles as straight lines homogeneously arranged along the muscle length, and/or their code is not readily accessible to the general public (not free and open source).

\section{Methodology}

For the purpose of this "proof of concept" communication, we used panoramic images, made of stitched B-mode scans of the vastus lateralis muscle. The analysis programme was designed to process images only depicting the thickness of one muscle, as defined by its shallow and deep aponeuroses (Figure 1). The Radon transforms of the upper and lower half of this image are then used to find first order line-approximations of the aponeuroses. Detected lines are divided into overlapping regions of interest (ROI), and the strongest ROIs are used as the starting points for segmenting the rest of the path. By applying

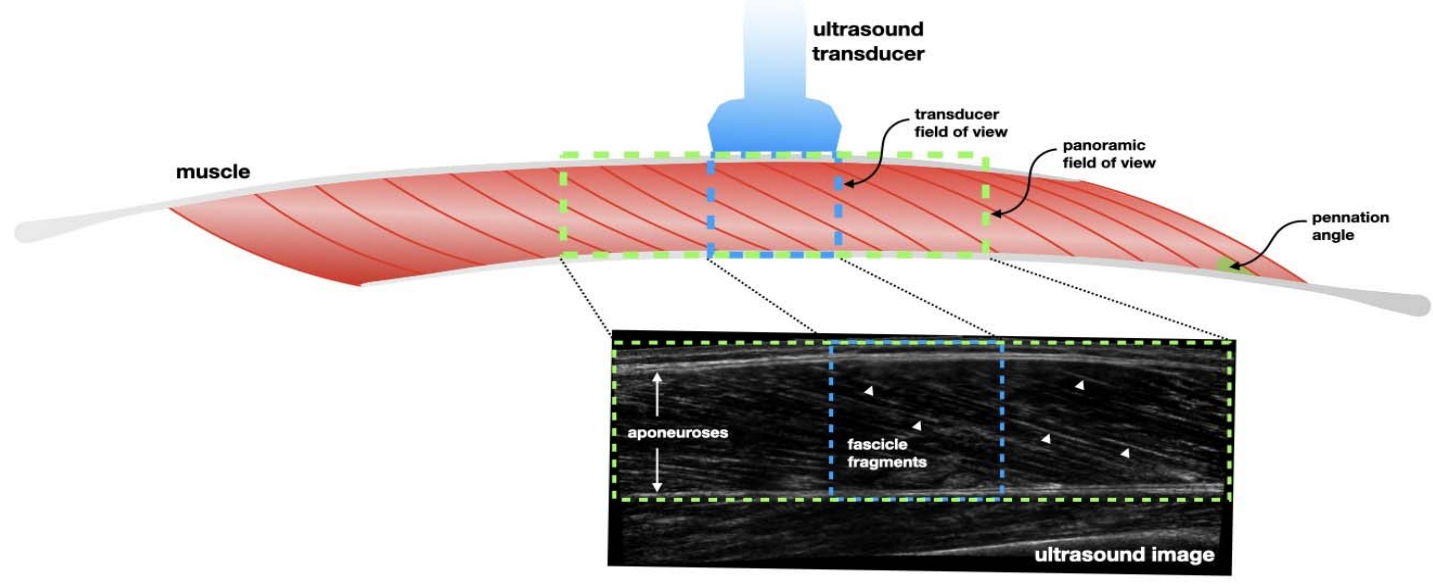

Figure 1 Schematic illustration of muscle imaging using ultrasound. 
a continuity condition, smooth aponeuroses outlines are found similar to the expected biological structures.

Fascicles are enhanced by applying a Frangi vesselness filter. Fascicle paths are found by measuring the dominant direction around a point through a large number of local radon transforms, weighted by the ratio of the median value of each radon transform, and divided by the corresponding variance. Based on the orientation of the line segment found in each ROI, contiguous ROIs are determined until the full reference fascicle has been characterized. The method and its GUI is made available in open source Python computer code [3], with the current GUI shown in Figure 2. The app can load DICOM, JPG and PNG image files, and utilizes standard Python packages such as NumPy and scikit-image.

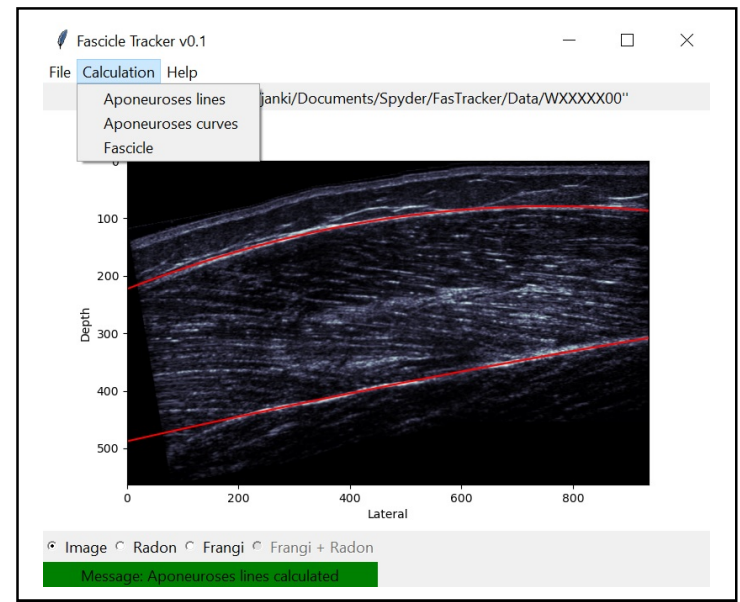

Figure 2 Open source FasTracker app made in Python.

\section{RESULTS}

The main features of interest for the fascicles are their length and pennation angle, i.e. the angle between the fascicle and the deep aponeuroses. As shown in Figure 3, consistent results were found for the pennation angles (ranging from $27.9^{\circ}$ to, $36.9^{\circ}$ ) and fascicle lengths (ranging from 8.1 to 9.2 centimeters). The results reflect the regional variability of fascicles conformation along the muscle length as intended. Visual inspection confirms the suitability of the proposed method with images from several subjects, with various signal-to-noise ratio, artefacts and muscle architecture.

\section{CONCLUSION}

A fully automated and robust method that determines nonlinear aponeuroses and fascicles over entire panoramic images is implemented and made available in open source Python computer code. The proposed method gives promising results for a more accurate and detailed study of muscle architecture. Further studies are needed to validate it and to extend its application to ultrasound videos of contracting muscles.

\section{REFERENCES}

[1] Zhou, Guang-Quan, Phoebe Chan, and Yong-Ping Zheng. 'Automatic Measurement of Pennation Angle and Fascicle Length of Gastrocnemius Muscles Using Real-Time Ultrasound Imaging'. Ultrasonics, 2014.

[2] Seynnes, Olivier R., and Neil J. Cronin. 'Simple Muscle Architecture Analysis (SMA): An ImageJ Macro Tool to Automate Measurements in B-Mode Ultrasound Scans'. PloS One 15, no. 2 (2020): e0229034. https://doi.org/10.1371/journal.pone.0229034

[3] Fascicle Tracker open source Python program available at GitHub: https://github.com/uio-janki/fastracker

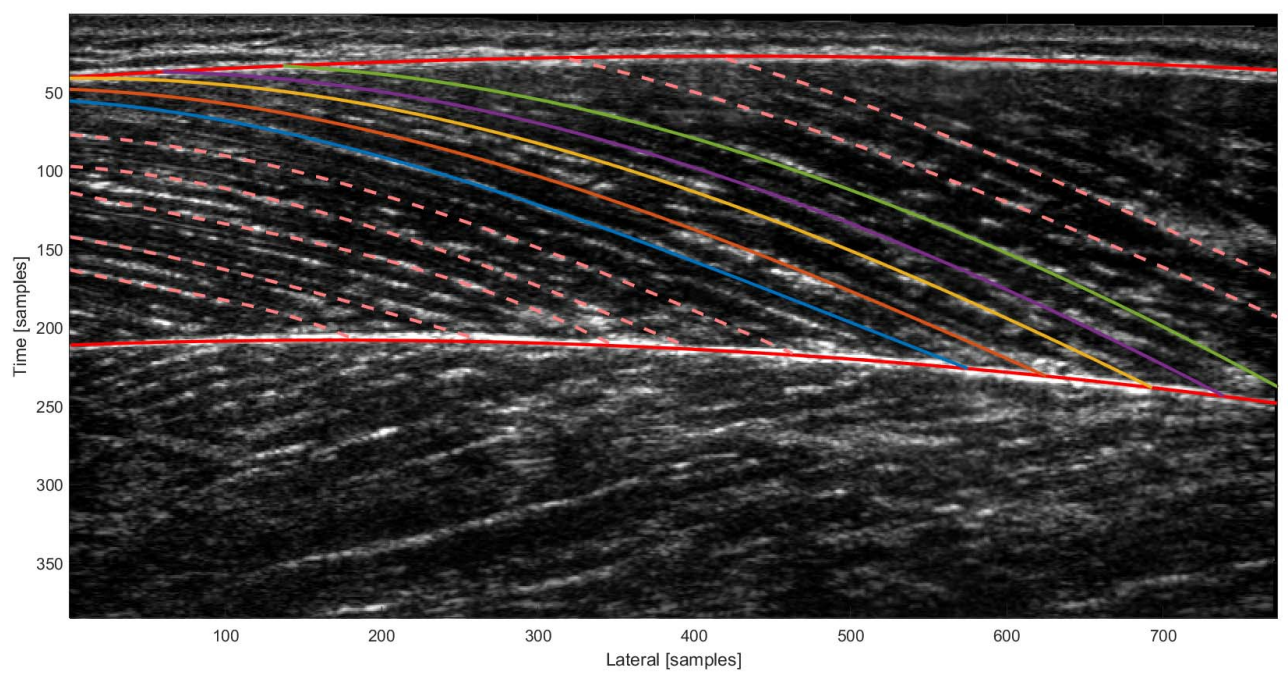

Figure 3 Ultrasound image of muscle, with shallow and deep aponeuroses (red lines) and five full-length reference fascicles. 